\title{
Alternativa Rápida e Barata para o Tratamento de Cromo Oriundo de Resíduos de DQO de Laboratórios de Estação de Tratamento Esgoto
}

\author{
Paola C. Oliveira \& Leandro M. Lourenço
}

Seguindo uma tendência mundial, a busca pela sustentabilidade no tratamento de resíduos químicos em universidades e centros de pesquisa vem ganhando força nos últimos anos. Uma das fontes de resíduo de metal pesado está na própria análise laboratorial da Demanda Química de Oxigênio (DQO), que é um parâmetro importante para a avaliação da concentração de contaminantes orgânicos nos recursos hídricos. O uso da DQO na caracterização de efluentes é discutido em termos da legislação vigente e seus métodos de determinação, colorimétricos e titulométricos, podem ser comparados estatisticamente. Atualmente, os testes de DQO, como alternativa aos testes de Demanda Bioquímica de Oxigênio, são amplamente utilizados em diversos municípios do Brasil e são suportados pela legislação vigente. Sabe-se que, em alguns municípios, a coleta destes resíduos se dá por empresas terceirizadas e, em outros, os testes são realizados sem nenhuma preocupação acerca da destinação dada aos resíduos produzidos, sendo muitas vezes despejados no esgoto. Entre os resíduos gerados, o menos perigoso é o ácido sulfúrico que pode ser neutralizado, nas quantidades e concentrações despejadas, não oferece maiores danos ao meio ambiente, porém, tem-se a presença dos metais pesados prata, cromo e mercúrio, altamente perigosos e cancerígenos. A fim de buscar uma alternativa aos métodos de tratamento de resíduos empregados, e numa tentativa de prestar suporte e assessoria à Estação de Tratamento de Esgoto do Município de Volta Redonda, o presente trabalho buscará investigar métodos alternativos, rápidos e baratos para o tratamento dos efluentes da análise de DQO.

\section{Palavras-chave: Resíduos Químicos; Tratamento de Resíduos; DQO, Metais Pesados.}

Following a worldwide trend, the pursuit of sustainability in the treatment of chemical residues in universities and research centers has been gaining strength in recent years. One of the sources of heavy metal residue is in the laboratory analysis of the chemical oxygen demand (COD), which is an important parameter for evaluating the concentration of organic contaminants in water resources. The use of cod in the characterisation of effluents is discussed in terms of current legislation and its methods of determination, colorimetric and volumetric, can be statistically compared. Currently, COD tests, as an alternative to the biochemical oxygen demand tests, are widely used in several municipalities in Brazil and are supported by the current legislation. It is known that, in some municipalities, the collection of these waste is given by outsourcing companies and, in others, the tests are conducted without any concern about the destination given to waste produced, being often dumped in the sewer. Among the generated residues, the less dangerous is sulfuric acid which, in the quantities and concentrations dumped, does not offer any greater damage to the environment, however, the presence of heavy metals silver, chromium and mercury, highly hazardous and carcinogenic. In order to seek an alternative to the methods of treatment of waste employed, and in an attempt to provide support and advice to the sewage treatment plant of Volta Redonda, this work will seek to investigate alternative, rapid and inexpensive methods for the treatment of effluents from cod analysis.

Keywords: Chemical Residues; Waste Treatment; COD; Heavy metals. 


\section{Introdução}

Seguindo uma tendência mundial, a busca pela sustentabilidade no tratamento de resíduos químicos em universidades e centros de pesquisa vem ganhando força nos últimos anos. Uma das fontes de resíduo de metal pesado está na própria análise laboratorial da Demanda Química de Oxigênio (DQO), que é um parâmetro importante para a avaliação da concentração de contaminantes orgânicos nos recursos hídricos. O uso da DQO na caracterização de efluentes é discutido em termos da legislação vigente e seus métodos de determinação, colorimétricos e titulométricos, podem ser comparados estatisticamente. Atualmente, os testes de DQO, como alternativa aos testes de Demanda Bioquímica de Oxigênio, são amplamente utilizados em diversos municípios do Brasil e são suportados pela legislação vigente. Sabe-se que, em alguns municípios, a coleta destes resíduos se dá por empresas terceirizadas e, em outros, os testes são realizados sem nenhuma preocupação acerca da destinação dada aos resíduos produzidos, sendo muitas vezes despejados no esgoto sem qualquer tratamento. A DQO tem como princípio a reação química da matéria orgânica e demais substâncias passíveis de oxidação com $\mathrm{K}_{2} \mathrm{Cr}_{2} \mathrm{O}_{7}$ e $\mathrm{H}_{2} \mathrm{SO}_{4}$, podendo ser determinada pelo método titulométrico ou colorimétrico. No método colorimétrico por refluxo fechado, de onde provém os resíduos tratados, normalmente utilizam-se os reagentes $\mathrm{AgSO}_{4}, \mathrm{HgSO}_{4}$, além do $\mathrm{H}_{2} \mathrm{SO}_{4}$ e $\mathrm{K}_{2} \mathrm{Cr}_{2} \mathrm{O}_{7}$ citados acima, juntamente com $2 \mathrm{~mL}$ de solução contendo o afluente(entrada) e o efluente(saída) da estação de tratamento de esgoto em vials diferentes, que são levados a digestão por duas horas a $150{ }^{\circ} \mathrm{C} .{ }^{5}$ Este método consiste, basicamente, na absorção de radiação ultravioleta e visível por espécies iônicas e moleculares em solução, compreendendo, assim, a análise colorimétrica visual e a espectrofotométrica, sendo esta última, a que oferece resultados mais confiáveis. Por meio desta técnica, é medida a absorção do dicromato residual no frasco de reação em um comprimento de onda determinado. Entre os resíduos gerados pelas estações de tratamento, o ácido sulfúrico pode ser facilmente neutralizado, porém, tem-se a presença dos metais pesados pra, cromo e mercúrio, que são cancerígenos. Sabendo-se disto, buscou-se neste trabalho verificar a eficiência da água oxigenada como alternativa rápida e barata para o tratamento de cromo destes efluentes. Para isso, utilizou-se a espectrofometria UV/VIS como técnica de quantificação de cromo no efluente tratado.

\section{Resultados e Discussão}

A fim de comprovar a eficácia do método de separação proposto, foram efetuados testes prévios por meio da semimicroanálise qualitativa nas soluções dos metais a serem identificados posteriormente. Seguiu-se a marcha analítica e utilizou-se sulfeto e iodeto como agentes redutores de cromo, conforme a Figura $1^{2}$

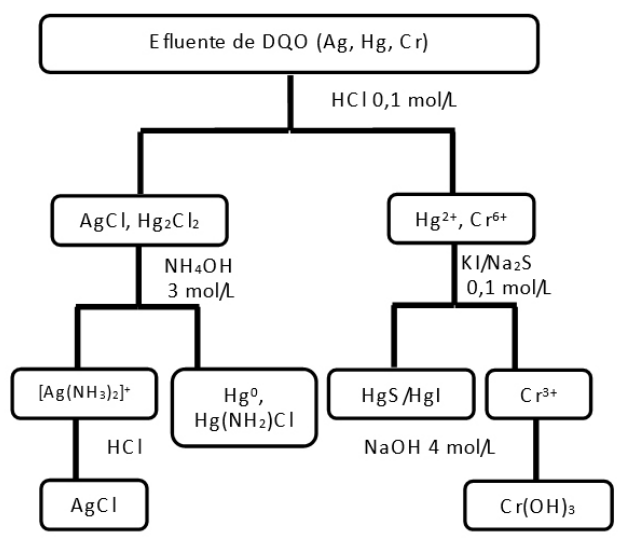

Figura 1. Separação por marcha analítica

Uma vez comprovada a eficácia do método de separação e, partindo da ideia de reduzir íons $\mathrm{Cr}^{6+}$ residuais em solução a $\mathrm{Cr}^{3+}$ por meio da água oxigenada, analisou-se uma amostra proveniente de um kit comercial comumente utilizado em estações de tratamento. Esta amostra contém a mistura de reagentes de DQO de uma estação de tratamento de água e esgoto antes da adição da matéria orgânica, ou seja, com a concentração máxima possível da espécie oxidante $\mathrm{Cr}^{6+}$. Fez-se isso para que se pudesse estimar a concentração de cromo presente nesta amostra comercial. Por meio de uma curva de calibração no intervalo de concentração de 0,04 a $0,1 \mathrm{~mol} / \mathrm{L}$, foi possível determinar 
a concentração presente em cada vial de $10 \mathrm{~mL}$ usado nas análises de DQO1, fornecendo como resultado um valor de concentração de $44 \mathrm{mg} / \mathrm{L}$ de cromo. Após separação analítica por meio das solubilidades das espécies e posterior redução do cromo com sulfeto e iodeto, que seguiu àquela demonstrada na Figura 1, as espécies foram quantificadas por espectrofotometria UV/VIS a $540 \mathrm{~nm}$. Os resultados obtidos, pós-tratamento, estão dispostos na Tabela 1 . É importante salientar que as concentrações máximas permitidas pelo CONAMA 430/2011 nos efluentes para o cromo hexavalente e trivalente são de 0,1 e $1,0 \mathrm{mg} / \mathrm{L}$, respectivamente. ${ }^{3}$

Tabela 1. Concentração de cromo pós-tratamento.

\begin{tabular}{|c|c|c|c|}
\hline Espécie & Precipitante & $\mathrm{C}(\mathrm{mg} / \mathrm{L})$ & $\begin{array}{c}\text { Frasco } \\
(\mathrm{mg} / 10 \mathrm{~mL})\end{array}$ \\
\hline $\mathrm{Cr}^{3+}$ & Iodeto & $1,2 \times 10-6$ & $1,2 \times 10-8$ \\
\hline $\mathrm{Cr}^{3+}$ & Sulfeto & 0,282 & 0,00282 \\
\hline $\mathrm{Cr} 2 \mathrm{O} 72-$ & Iodeto & 388 & 3,88 \\
\hline $\mathrm{Cr} 2 \mathrm{O} 72-$ & Sulfeto & 1680 & 16,80 \\
\hline $\mathrm{Cr}^{6+}(*)$ & Iodeto & 308 & 3,08 \\
\hline $\mathrm{Cr}^{6+}(*)$ & Sulfeto & 1333,92 & 13,3392 \\
\hline
\end{tabular}

*refere-se à concentração de $\mathrm{Cr}^{6+}$ no $\mathrm{Cr}_{2} \mathrm{O}_{7}^{2-}$

Pela Tabela 1, nota-se que o iodeto é o melhor agente redutor em termos de resíduos de cromo pós-tratamento. Observa-se ainda que as concentrações de Cr6+ permaneceram acima dos valores máximos permitidos pela legislação. Como alternativa, optou-se por reduzir o cromo residual com $\mathrm{H} 2 \mathrm{O} 2$, um redutor reconhecidamente limpo, pois gera como outros produtos de reação apenas $\mathrm{O} 2$ e $\mathrm{H} 2 \mathrm{O}$, conforme reação química abaixo.

$$
\mathrm{Cr}_{2} \mathrm{O}_{7}^{2-}+3 \mathrm{H}_{2} \mathrm{O}_{2} \rightarrow 2 \mathrm{Cr}^{3+}+7 \mathrm{H}_{2} \mathrm{O}+3 \mathrm{O}_{2}
$$

Os testes de eficácia foram confirmados pela presença dos picos característicos das espécies trivalente (410 e 570 $\mathrm{nm})$ e hexavalente $(380 \mathrm{~nm})$ de cromo. Testando a eficácia de acordo com a variação de condições de análise, variou- se o volume de $\mathrm{H}_{2} \mathrm{O}_{2}$ adicionado e $\mathrm{o} \mathrm{pH}$ por meio da adição de $\mathrm{H}_{2} \mathrm{SO}_{4}$. Os melhores resultados foram alcançados em um valor de $\mathrm{pH}$ igual a 2 com adição de $2 \mathrm{~mL}$ de $\mathrm{H}_{2} \mathrm{O}_{2} 14$ volumes para cada vial de $10 \mathrm{~mL}$. $^{4-5}$

Tabela 2. Efeito do $\mathrm{pH}$ e variação do volume de $\mathrm{H}_{2} \mathrm{O}_{2}$

\begin{tabular}{|c|c|c|c|}
\hline$\lambda(\mathrm{nm})$ & $\mathrm{A} 1 *$ & $\mathrm{~A} 2 \#$ & $\mathrm{~A} 3 \%$ \\
\hline 380 & 0,713 & 0,337 & - \\
\hline 410 & 0,160 & 0,273 & 1,245 \\
\hline 570 & 0,089 & 0,072 & 1,902 \\
\hline
\end{tabular}

(*) $\mathrm{pH}=1 ; 0,15 \mathrm{~mL} \mathrm{H} 2 \mathrm{O} 2$; (\#) $\mathrm{pH}=2 ; 0,3 \mathrm{~mL} \mathrm{H} 2 \mathrm{O} 2$

(\%) $\mathrm{pH}=2 ; 2 \mathrm{~mL} \mathrm{H} 2 \mathrm{O} 2$

Os testes com as soluções padrão foram realizados de forma qualitativa, e se mostraram de fácil aplicação e com curto tempo de reação (aproximadamente 2 minutos). Para que este método proposto seja aplicado de maneira eficaz em estações de tratamento de água e esgoto, propõe-se inicialmente a separação dos metais pela marcha analítica (Figura 1) seguida do tratamento do resíduo de cromo. Após a primeira leitura de teor residual, se este estiver acima do permitido em legislação, deve-se adicionar $\mathrm{H} 2 \mathrm{O} 2$ para reduzir o restante do cromo até que os valores atendam àqueles indicados na lei.

Por fim, avaliou-se os custos de reagentes para o tratamento proposto em uma estação de médio porte como a do município de Volta Redonda - RJ, e foi estimado um custo de análise anual de $\mathrm{R} \$ 480,00$.

\section{Conclusões}

A redução de cromo com $\mathrm{H}_{2} \mathrm{O}_{2}$ mostra-se eficaz e relativamente barata, produzindo efluentes que atendem aos níveis máximos permitidos em legislação. Outra vantagem no uso da $\mathrm{H}_{2} \mathrm{O}_{2}$, é que se trata de um reagente que minimiza a produção de novos resíduos químicos. Percebe-se assim a importância, não só ambiental, como também orçamentária de um programa responsável de tratamento e gestão de resíduos. É desejável que programas assim sejam bem elaborados e, se corretamente executados, auxiliarão muitos municípios a atender os índices preconizados pela lei. Este 


\section{Artigo Geral 24}

programa deve ser constantemente revisado, reformulado e avaliado quanto à sua correta aplicação e eficácia.

\section{Agradecimentos}

Os autores deste trabalho agradecem à Universidade Federal Fluminense - UFF e ao Serviço Autônomo de Água e Esgoto de Volta Redonda (SAAE-VR).

\section{Referências}

1. Lauffer, J.; Chernicharo, C. A. De L.; Keller, W.; H. Pos, W. Análise Crítica das metodologias colorimétrica e titulométrica para determinação da demanda química de oxigênio em amostras de esgotos sanitários. In: 20o CONGRESSO BRASILEIRO DE ENGENHARIA SANITÁRIA E AMBIENTAL, ABES - Associação Brasileira de Engenharia Sanitária e Ambiental.

2. Vogel, A. I. Química Analítica Qualitativa. São Paulo: Mestre Jou, 1981. $665 \mathrm{p}$

3. Brasil. Resolução CONAMA N ${ }^{\circ} 430$ de 13 de maio de 2011. Dispõe sobre condições e padrões de lançamento de efluentes, complementa e altera a Resolução n ${ }^{\circ}$ 357, de 17 de março de 2005, do Conselho Nacional do Meio Ambiente - CONAMA.

4. Reis, M. X.; Teixeira, L. A. C. Remoção de cromo de efluentes por redução com peróxido de hidrogênio e precipitação de hidróxido. In: VI SHMMT I XVIII ENTMME - 2001, Rio de Janeiro/Brasil, 2001.

5. Standard Methods for the examination of water and wastewater. American Public Health Association, American Water Works Association, Water Evironmental Federation, 20th ed. Washington

\section{Paola C. Oliveira* \& Leandro M. Lourenço}

Departamento de Química, Instituto de Ciências Exatas - Universidade Federal Fluminense - Campus Aterrado CEP 27213-145 - Volta Redonda - RJ, Brasil

"E-mail: paolaoliveira@id.uff.br 